\title{
Composite Fermions and the Energy Gap in the Fractional Quantum Hall Effect
}

\author{
N. E. Bonesteel \\ National High Magnetic Field Laboratory, Florida State University, Tallahassee, FL 32306-4005
}

(August 8, 2018)

\begin{abstract}
The energy gaps for the fractional quantum Hall effect at filling fractions $1 / 3,1 / 5$, and $1 / 7$ have been calculated by variational Monte Carlo using Jain's composite fermion wave functions before and after projection onto the lowest Landau level. Before projection there is a contribution to the energy gaps from the first excited Landau level. After projection this contribution vanishes, the quasielectron charge becomes more localized, and the Coulomb energy contribution increases. The projected gaps agree well with previous calculations, lending support to the composite fermion theory.
\end{abstract}

Typeset using REVTEX 
In both the integer and fractional quantum Hall effect (IQHE and FQHE) a two dimensional electron gas in a strong perpendicular magnetic field $B$ exhibits a precisely quantized Hall resistance and an exponentially activated longitudinal resistance $R_{x x} \propto \exp -\Delta / 2 K T$ indicating the appearance of an energy gap $\Delta$ in the system $1 \mathbb{1}[3]$. In the IQHE $\Delta$ is equal to the energy required to promote an electron from the highest filled Landau level to the lowest empty Landau level, which in a weakly disordered system with fully polarized spins is approximately the cyclotron energy: $\Delta \simeq \hbar \omega_{c} \equiv \hbar e B / m^{*} c$ where $m^{*}$ is the band mass of the electron. This is to be contrasted with the FQHE where an energy gap forms in a partially filled Landau level due to correlation effects [4] and $\Delta \propto e^{2} / \epsilon l_{0}$ where $\epsilon$ is the dielectric constant and $l_{0}=\sqrt{\hbar c / e B}$ is the magnetic length.

Jain has introduced the concept of composite fermions, electrons bound to an even number of flux quanta, in order to treat the IQHE and FQHE on the same footing [5]. According to Jain's theory composite fermions experience an effective magnetic field $B_{\text {eff }}=$ $B-2 k \phi_{0} n$ where $\phi_{0}=h c / e$ is the flux quantum, $2 k$ is the number of bound flux quanta, and $n$ is the electron density. When composite fermions fill $p$ pseudo-Landau levels, where a pseudo-Landau level is a Landau level corresponding to the effective magnetic field $B_{\text {eff }}$, the physical Landau level filling fraction is $\nu=p /(2 k p+1)$ and the system exhibits a FQHE with $\Delta$ equal to the energy required to promote a composite fermion from the $p$ th to the $(p+1)$ th pseudo-Landau level. The energy gap for the FQHE can therefore be thought of as an effective cyclotron energy for composite fermions. Du et al. [6] have measured the energy gaps for the sequence $\nu=p /(2 p+1)$ and found that $\Delta(B) \propto\left|B_{\text {eff }}\right|=\left|B-B_{1 / 2}\right|$ where $B_{1 / 2}=2 \phi_{o} n$, consistent with Jain's theory [0]. In addition, a number of experiments exploring the regime near $\nu \sim 1 / 2$ where $B_{\text {eff }} \sim 0$ have provided evidence for the existence of a composite fermion 'metal' in this limit [8,9]. These experiments have led to a renewed interest in composite fermions.

In this paper I present calculations of the energy gaps for the $\nu=1 / 3,1 / 5$, and $1 / 7$ FQHE using Jain's composite fermion wave functions [5]. Before proceeding it is useful to contrast the present work with that of Morf and Halperin [10] who calculated $\Delta$ for $\nu=1 / 3$ 
by first calculating the excitation energies for an isolated quasielectron $E_{\mathrm{qe}}$ and quasihole $E_{\mathrm{qh}}$ using Laughlin's trial wave functions in the disk geometry. Morf and Halperin found that $\Delta=E_{\mathrm{qe}}+E_{\mathrm{qh}} \simeq 0.1 e^{2} / \epsilon l_{0}$, a result which agrees well with experiment once the effects of Landau level mixing [11] and the finite thickness of the wave function [12] are properly taken into account [13]. Note that although the quasielectron and quasihole form a bound state (the magnetoroton) with lower energy than $\Delta$ this state does not carry current and so does not contribute to $R_{x x}$. In Jain's theory the ground state for $\nu=1 /(2 k+1)$ is obtained by completely filling the lowest pseudo-Landau level with composite fermions. A quasihole is created by removing a composite fermion from this state, while a quasielectron is created by adding a composite fermion to the first excited pseudo-Landau level. Jain has shown that the composite fermion wave functions for the ground state and quasihole state are identical to Laughlin's [5]. Thus the essential difference between the present calculation and that of Morf and Halperin is that Jain's rather than Laughlin's quasielectron wave function is used.

Because Jain's quasielectron wave function has a nonzero overlap with the first excited Landau level it is necessary to project onto the lowest Landau level to study the $B \rightarrow$ $\infty$ limit. Previously this has only been done on small systems (up to 10 electrons for $\nu=1 / 3)$ where it is possible to perform this projection exactly [14]. Here it is shown how projected expectation values for states with a single quasielectron can be expressed in terms of unprojected expectation values which can then be evaluated by variational Monte Carlo. Using this projection technique it is possible to study larger systems than have previously been studied and perform a reliable extrapolation to the thermodynamic limit. The main result of this paper is that for $\nu=1 /(2 k+1)$ projection not only trivially ensures that the contribution to the energy gap from the first excited Landau level vanishes, but also has the effect of further localizing the charge of the quasielectron, thus transforming the kinetic energy contribution to the unprojected energy gap $\left(\propto \hbar \omega_{c}\right)$ into an increased Coulomb contribution $\left(\propto e^{2} / \epsilon l_{0}\right)$. For $\nu=1 / 3$ and $1 / 5$ the projected energy gaps agree well with previous calculations, establishing the variational validity of Jain's quasielectron wave function. This is a nontrivial result because even after projection Jain's quasielectron is not 
equivalent to Laughlin's [5].

Adopting the spherical geometry introduced by Haldane 15 the Hamiltonian for spin polarized electrons on a sphere with a magnetic monopole at the center is $H=T+V$ where

$$
T=\frac{\omega_{c}}{2 \hbar S} \sum_{i=1}^{N}\left(\mathbf{r}_{i} \times\left(i \hbar \nabla_{i}-\frac{e}{c} \mathbf{a}_{i}\right)\right)^{2}-\frac{N}{2} \hbar \omega_{c}
$$

and

$$
V=\sum_{i<j} \frac{e^{2}}{\epsilon r_{i j}} .
$$

Here $2 S$ is the number of flux quanta passing through the surface of the sphere, $N$ is the total number of electrons, $\mathbf{a}_{i}=S \mathbf{e}_{\phi} \cot \theta_{i} / R$ is the vector potential corresponding to the magnetic field $B=2 S \phi_{0} / 4 \pi R^{2}$, and $R$ is the radius of the sphere. The zero point kinetic energy has been subtracted from $T$ and the distance between electrons in $V$ is taken to be the chord distance. The one-body eigenstates of $T$ in the lowest and first excited Landau levels with eigenvalues $E_{0}=0$ and $E_{1}=(1+1 / S) \hbar \omega_{c}$ have angular momentum $l=S$ and $l=S+1$ and are given by

$$
\Upsilon_{S, m} \propto u^{S-m} v^{S+m}
$$

for $m=-S, \ldots, S$ and

$$
\Upsilon_{S+1, m} \propto((2 S+2) \bar{v} v+m+S+1) u^{S-m} v^{S+m}
$$

for $m=-S-1, \ldots, S+1$ [15]. Here $m$ labels the $z$ component of the angular momentum, and $u=\cos \theta / 2 \exp -i \phi / 2$ and $v=\sin \theta / 2 \exp i \phi / 2$ are the spinor coordinates of the electron.

Jain has proposed a simple relationship between the physical electron wave function $\psi$ and the corresponding 'mean field' composite fermion wave function $\Phi_{\mathrm{CF}}$. On the sphere this relationship is

$$
\psi=\prod_{i<j}\left(u_{i} v_{j}-v_{i} u_{j}\right)^{2 k} \Phi_{\mathrm{CF}}
$$

where the Jastrow factor in (5) corresponds intuitively to attaching $2 k$ flux quanta to the electrons and can be derived microscopically using the Chern-Simons theory of composite fermions [16]. 
Using (5) it is straightforward to construct the ground state and excited state wave functions needed to calculate $\Delta$. When $\nu=1 /(2 k+1)$ the number of flux quanta passing through the surface of the sphere is related to the total number of electrons by $2 S=$ $(2 k+1)(N-1)$ [15]. For this field strength the effective field seen by composite fermions with $2 k$ flux quanta attached corresponds to $2 S^{*}=N-1$ [5]. When the lowest pseudoLandau level is completely full the mean field composite fermion wave function is

$$
\begin{aligned}
\Phi_{C F} & =\left|\begin{array}{ccccc}
u_{1}^{N-1} & u_{1}^{N-2} v_{1} & \ldots & u_{1} v_{1}^{N-2} & v_{1}^{N-1} \\
\vdots & \vdots & & \vdots & \vdots \\
u_{N}^{N-1} & u_{N}^{N-2} v_{N} & \ldots & u_{N} v_{N}^{N-2} & v_{N}^{N-1}
\end{array}\right| \\
& =\prod_{i<j}\left(u_{i} v_{j}-v_{i} u_{j}\right)
\end{aligned}
$$

and the physical ground state wave function $\psi$ is precisely Laughlin's wave function [15].

A low energy band of excited states above the $\nu=1 /(2 k+1)$ ground state is constructed by promoting a composite fermion from the lowest pseudo-Landau level to the first excited pseudo-Landau level. On the sphere these excited states form multiplets labeled by their total angular momentum $l_{\text {tot }}=1, \ldots, N$. For small systems (up to 10 electrons) Dev and Jain [17] have shown that after projection the $l_{\text {tot }}=1$ state is eliminated and the remaining band of low energy states have large overlaps with the corresponding eigenstates obtained by exact diagonalization. Here I concentrate on the $l_{\text {tot }}=N$ multiplet. The trial state for the $m_{\text {tot }}=-N$ member of this multiplet is formed by removing a composite fermion from the lowest pseudo-Landau level at the bottom of the sphere and reintroducing it into the first excited pseudo-Landau level at the top of the sphere. The mean field composite fermion wave function for this state is

$$
\Phi_{C F}^{\prime}=\left|\begin{array}{ccccc}
u_{1}^{N-1} & u_{1}^{N-2} v_{1} & \ldots & u_{1} v_{1}^{N-2} & u_{1}^{N-1} \bar{v}_{1} \\
\vdots & \vdots & & \vdots & \vdots \\
u_{N}^{N-1} & u_{N}^{N-2} v_{1} & \ldots & u_{N} v_{N}^{N-2} & u_{N}^{N-1} \bar{v}_{N}
\end{array}\right|
$$

and the corresponding physical wave function $\psi^{\prime}$ describes a state with a quasielectron at the top of the sphere and a quasihole at the bottom of the sphere. In the $N \rightarrow \infty$ limit the 
quasielectron and quasihole are infinitely separated and the excitation energy of this state should therefore correspond to the energy gap $\Delta$ which appears in the activated temperature dependence of $R_{x x}$.

Before discussing the calculation of the energy gaps it is necessary to address the problem of projecting $\psi^{\prime}$ onto the lowest Landau level. Because $\psi^{\prime}$ contains only a single composite fermion in the first excited pseudo-Landau level it can be decomposed as

$$
\psi^{\prime}=\psi_{0}^{\prime}+\psi_{1}^{\prime}
$$

where $\psi_{0}^{\prime}$ is the projected state with all $N$ electrons in the lowest Landau level and $\psi_{1}^{\prime}$ is orthogonal to $\psi_{0}^{\prime}$ and has $N-1$ electrons in the lowest Landau level and 1 electron in the first excited Landau level. It follows that $T \psi_{0}^{\prime}=0$ and $T \psi_{1}^{\prime}=E_{1} \psi_{1}^{\prime}$ which implies

$$
\psi_{0}^{\prime} \propto\left(T-E_{1}\right) \psi^{\prime}
$$

The projected expectation value of a given operator $O$ can now be expressed in terms of unprojected expectation values as

$$
\langle O\rangle_{\text {proj. }}=\frac{\left\langle\left(T-E_{1}\right) O\left(T-E_{1}\right)\right\rangle}{\left\langle\left(T-E_{1}\right)^{2}\right\rangle} .
$$

Equation (10) is ideally suited for evaluation by variational Monte Carlo. The inspiration for this technique is the generalized Lanczos method of Heeb and Rice [18 for systematically improving a variational wave function by applying a local operator, not necessarily the Hamiltonian, to that wave function. Here $\psi^{\prime}$ contains only a single quasielectron and so one iteration with $T-E_{1}$ is sufficient to do the full projection. However, in principle this method can be applied to the FQHE hierarchy $(p>1)$ where it would provide a systematic method for improving Jain's composite fermion wave functions.

Figure 1 shows the density profile of the excited state wave functions $\psi^{\prime}$ with 12 electrons for $\nu=1 / 3,1 / 5$, and $1 / 7$ before and after projection onto the lowest Landau level. In each case the charge deficit at the bottom of the sphere $(\theta=\pi)$ is a quasihole, while the excess charge localized in a ring near the top of the sphere $(\theta=0)$ is a quasielectron. While the 
quasihole is unaffected by projection because it is entirely in the lowest Landau level, the quasielectron charge becomes more localized after projection. This is a natural result given that the unprojected wave function has more degrees of freedom than the projected wave function. Note that the effect of projection on the quasielectron grows weaker as $\nu$ decreases reflecting the fact that the Jastrow factor in (5) becomes more effective at projecting into the lowest Landau level with increasing $k$ [5].

Figure 2 shows the kinetic $(T)$ and Coulomb $(V)$ contributions to $\Delta$ before and after projection onto the lowest Landau level plotted vs. $1 / N$ for $\nu=1 / 3$. A trivial systematic size dependence of the Coulomb contribution has been removed by subtracting out the Coulomb energy of two point charges with fractional charge $\pm e /(2 k+1)$ at the top and bottom of the sphere,

$$
\Delta_{\text {Coul. }}=\langle V\rangle^{\prime}-\langle V\rangle+\frac{(e /(2 k+1))^{2}}{2 \epsilon R}
$$

Likewise, the kinetic energy contribution has been modified to account for the size dependence of the energy difference between the lowest and first excited pseudo-Landau levels,

$$
\Delta_{\text {K.E. }}=\left(\langle T\rangle^{\prime}-\langle T\rangle\right) /\left(1+\frac{1}{S^{*}}\right)
$$

Here $\langle\ldots\rangle$ and $\langle\ldots\rangle^{\prime}$ denote expectation values (either projected or unprojected) in $\psi$ and $\psi^{\prime}$. As can be seen in Fig. 2 the size dependence of the corrected energy gaps is small for large enough $N$.

It is now possible to summarize the effect of Landau level projection on the energy gap. For $\nu=1 / 3$, before projection, $\Delta=\Delta_{\text {Coul. }}+\Delta_{\text {K.E. }} \simeq 0.05 e^{2} / \epsilon l_{0}+0.16 \hbar \omega_{c}$, consistent with the calculations of Trivedi and Jain [19]. This result is clearly unphysical in the extreme quantum limit where the only energy scale in the problem is the Coulomb energy $\sim e^{2} / \epsilon l_{0}$. After projection $\Delta_{\text {K.E. }}=0$ and, as shown above, the quasielectron charge has become more localized. This in turn leads to an increase in the Coulomb energy of the quasielectron yielding the projected energy gap $\Delta \simeq 0.1 e^{2} / \epsilon l_{0}$, consistent both with Morf and Halperin's variational calculation [10] and with exact diagonalization studies of small systems [20,21]. 
The results of similar calculations for $\nu=1 / 5$ and $\nu=1 / 7$ are summarized in Table 1 . As for $\nu=1 / 3$ projection removes the kinetic energy contribution to $\Delta\left(\propto \hbar \omega_{c}\right)$ while concentrating the quasielectron charge and increasing the Coulomb contribution $\left(\propto e^{2} / \epsilon l_{0}\right)$. Table 1 also includes the exact diagonalization results of Fano et al. [21] for the energy gap extrapolated to infinite system size for $\nu=1 / 3$ (up to 10 electrons) and $\nu=1 / 5$ (up to 7 electrons). The agreement between these energy gaps and those obtained here demonstrates the variational validity of Jain's quasielectron wave function for $\nu=1 / 3$ and $1 / 5$. Note that because the size of the Hilbert space needed to study systems with a given number of electrons grows factorially with $k$, the method used here is the only efficient way to calculate properties of projected composite fermion wave functions, even with a single quasielectron, for $\nu=1 / 7$.

To conclude, the energy gaps for the $\nu=1 / 3,1 / 5$, and $1 / 7$ FQHE have been calculated by variational Monte Carlo using Jain's composite fermion wave functions. Results have been obtained before and after projection onto the lowest Landau level using a novel projection technique. Before projection a significant contribution to the energy gap comes from the first excited Landau level. After projection this contribution vanishes and the charge of the quasielectron becomes more localized leading to an increased Coulomb contribution to the energy gap. For $\nu=1 / 3$ and $1 / 5$ the projected energy gaps agree well with previous calculations based on Laughlin's quasielectron wave function and exact diagonalization studies of small systems. This agreement establishes the variational validity of Jain's quasielectron wave function and lends support to the composite fermion theory. Clearly it would be interesting to generalize the projection technique introduced in this paper to study the FQHE hierarchy.

I would like to acknowledge useful discussions with N. Trivedi, E. Heeb, C.S. Hellberg, T.-L. Ho, and J.R. Schrieffer. This work was supported by NSF grant No. DMR-92-22682 and by the National High Magnetic Field Laboratory at Florida State University. 


\section{REFERENCES}

[1] K. von Klitzing, G. Dorda, and M. Pepper, Phys. Rev. Lett. 45, 494 (1980).

[2] D.C. Tsui, H.L. Stormer, and A.C. Gossard, Phys. Rev. Lett. 48, 1559 (1982).

[3] For a review see The Quantum Hall Effect, edited by R.E. Prange and S.M. Girvin (Springer-Verlag, New York, 1990).

[4] R.B. Laughlin, Phys. Rev. Lett. 50, 1395 (1983).

[5] J.K. Jain, Phys. Rev. Lett. 63, 199 (1989); Phys. Rev. B 40, 8079 (1989); 41, 7653 (1990).

[6] R.R. Du, H.L. Stormer, D.C. Tsui, L.N. Pfeiffer, and K.W. West, Phys. Rev. Lett. 70, 2944 (1992).

[7] More precisely Du et al. found that $\Delta=a|B|-\Gamma$ and interpreted $\Gamma$ as a broadening of the pseudo-Landau levels by impurity scattering.

[8] W. Kang, H.L. Stormer, L.N. Pfeiffer, K.W. Baldwin, and K.W. West, Phys. Rev. Lett. 71, 3850 (1993); R.L. Willett, R.R. Ruel, K.W. West, and L.N. Pfeiffer, Phys. Rev. Lett. 71, 3846 (1993).

[9] B.I. Halperin, P.A. Lee, and N. Read, Phys. Rev. B 47, 7312 (1993); V. Kalmeyer and S.C. Zhang, Phys. Rev. B 46, 9889 (1992).

[10] R. Morf and B.I. Halperin, Phys. Rev. B 33, 2221 (1986).

[11] D. Yoshioka, J. Phys. Soc. Jpn. 55, 885 (1986).

[12] F.C. Zhang and S. Das Sarma, Phys. Rev. B 31, 5280 (1986).

[13] R.L. Willett, H.L. Stormer, D.C. Tsui, A.C. Gossard, and J.H. English, Phys. Rev. B 37, 8476 (1988).

[14] M. Kasner and W. Apel, Phys. Rev. B 48, 11435 (1993).; U. Girlich and M. Hellmund, 
Preprint cond-mat/9403090.

[15] F.D.M. Haldane, Phys. Rev. Lett. 51, 605 (1983); and in [3.

[16] A. Lopez and E. Fradkin, Phys. Rev. B 44, 5246 (1991).

[17] G. Dev and J.K. Jain, Phys. Rev. Lett. 69, 2843 (1992).

[18] E.S. Heeb and T.M. Rice, Z. Phys. B 90, 73 (1993); and Preprint (cond-mat/9405039).

[19] N. Trivedi and J. Jain, Mod. Phys. Lett. B 5503 (1991).

[20] F.D.M. Haldane and E.H. Rezayi, Phys. Rev. Lett. 54, 237 (1985).

[21] G. Fano, F. Ortolani, and E. Columbo, Phys. Rev. B 34, 2670 (1986). 


\section{TABLES}

TABLE I. Unprojected and projected energy gaps for the FQHE with $\nu=1 / 3,1 / 5$, and $1 / 7$ calculated using Jain's composite fermion wave functions. The extrapolated exact diagonalization results of Fano et al. 21] are given for comparison. Variational Monte Carlo results are for 42 electrons.

\begin{tabular}{lcccc}
\hline \hline & $\Delta_{\text {K.E. }}$ & $\Delta_{\text {Coul. }}$ & $\Delta_{\text {Coul. }}$ & $\Delta$ \\
$\nu$ & $($ unprojected $)$ & (unprojected) & (projected) & (Ref. [21]) \\
\hline $1 / 3$ & $\left(\hbar \omega_{c}\right)$ & $\left(e^{2} / \epsilon l_{0}\right)$ & $\left(e^{2} / \epsilon l_{0}\right)$ & $\left(e^{2} / \epsilon l_{0}\right)$ \\
$1 / 5$ & $0.163(2)$ & $0.048(2)$ & $0.106(3)$ & $0.1036(2)$ \\
$1 / 7$ & $0.082(2)$ & $0.014(2)$ & $0.025(3)$ & $0.0244(3)$ \\
\hline \hline
\end{tabular}




\section{FIGURES}

FIG. 1. Density profile of the $l_{\text {tot }}=N, m_{\text {tot }}=-N$ excited state wave functions with $\nu=1 / 3$, $1 / 5$, and $1 / 7$ for a system with 12 electrons before and after projection onto the lowest Landau level. For each case the quasihole at the bottom of the sphere $(\theta=\pi)$ is unaffected by projection, while the quasielectron charge at the top of the sphere $(\theta=0)$ becomes more localized.

FIG. 2. Kinetic and Coulomb contributions to the energy gap vs. $1 / N$ for $\nu=1 / 3$ calculated using Jain's composite fermion wave functions before and after projection onto the lowest Landau level. Before projection $\Delta_{\text {K.E. }} \neq 0$ indicating a nonzero overlap with the first excited Landau level. After projection $\Delta_{\text {K.E. }}=0$ (not shown) and $\Delta_{\text {Coul. }}$ has increased because the quasielectron charge has become more localized. 

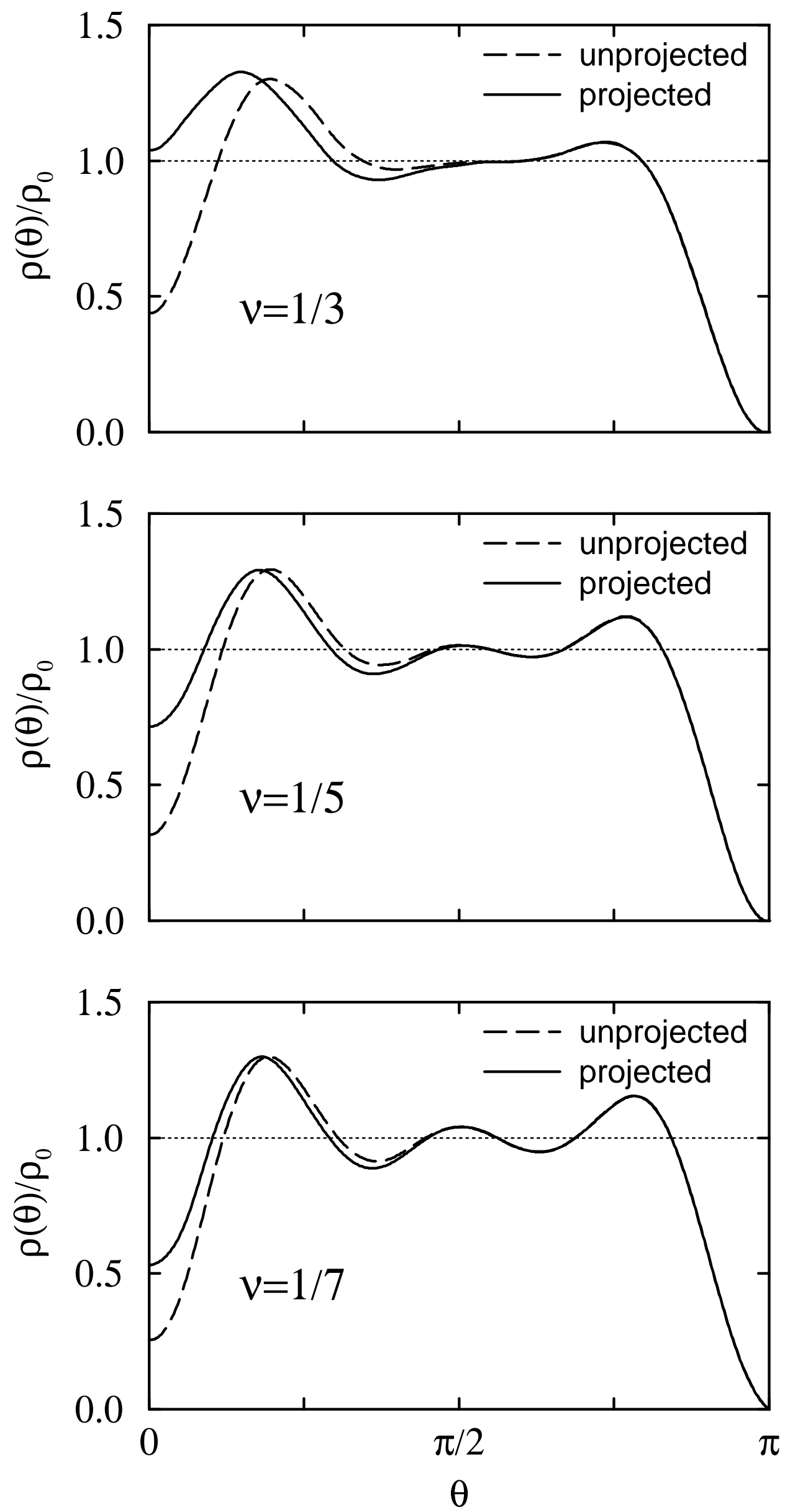

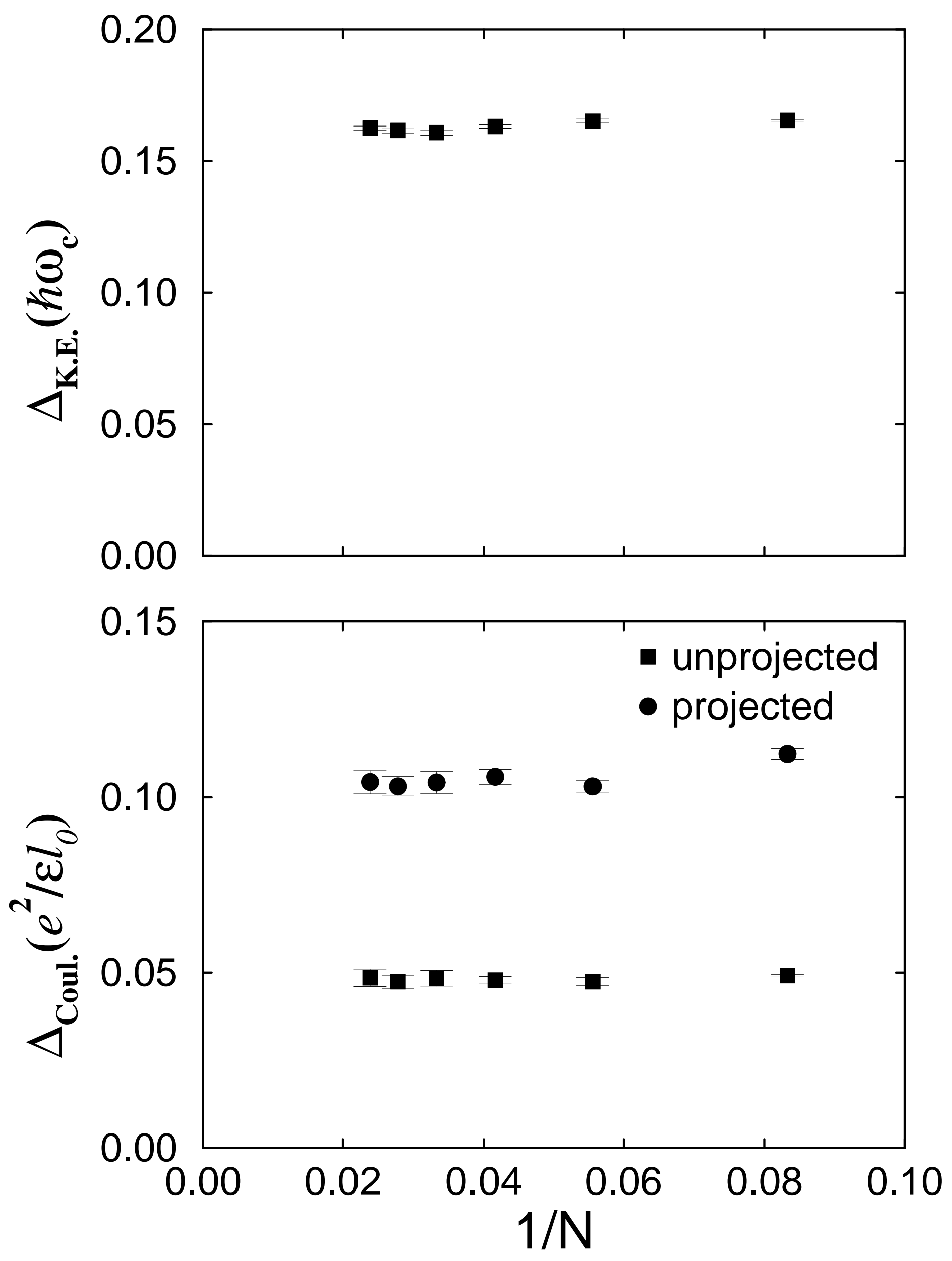